Case Report,

\title{
Diagnosis and Surgical Treatment of Esophageal Leiomyoma
}

\author{
Georgi Yankov, Evgeni Mekov ${ }^{1}$, Plamen Getsov ${ }^{2}$, Stanislav Churchev ${ }^{3}$, Branimir Golemanov ${ }^{3}$, Mila \\ Kovacheva-Slavova $^{3}$, Borislav Vladimirov ${ }^{3}$ \\ ${ }^{1}$ Department of pulmonary diseases, MBALBB "Saint Sofia", Medical University - Sofia, Bulgaria \\ ${ }^{2}$ Department of Radiology, University Hospital "Queen Joanna-ISUL", Medical University - Sofia, Bulgaria \\ ${ }^{3}$ Department of Gastroenterology, University Hospital "Queen Joanna-ISUL", Medical University - Sofia, \\ Bulgaria
}

\begin{abstract}
:
Leiomyomas are benign mesenchymal tumors, and together with gastrointestinal stromal tumors (GISTs), are the most common non-epithelial tumors originating from connective tissue in the deep layers of the esophagus. Histologically, both types of tumors differentiate based on immunohistochemical reactions. Very often, they are asymptomatic and are detected by chance using imaging methods and advanced endoscopy. The removal of the esophageal leiomyoma is performed through mini-invasive or open surgery. Herein, we present a patient diagnosed with esophageal leiomyoma located in the upper third of the esophagus by computered tomography and endoscopic ultrasound. Right-sided transthoracic extirpation of leiomyoma was performed.
\end{abstract}

Key words: Benign esophageal tumors; Leiomyoma; Esophagoscopic removal; Oesophageal enucleation

\section{Introduction:}

Leiomyomas are the most prevalent benign tumors of the esophagus and (after gists) are the second most common mesenchymal neoplasms of the gastrointestinal tract (GIT). Leiomyomas arise from the smooth muscle tissue in the esophageal wall usually from the muscularis propria but in some cases from the muscularis mucosae [1]. In the esophagus they are about three times more frequent than gists and can also be found in the colon, rectum, rarely in the stomach and are seldom observed in the small intestine. Autopsy series have demonstrated that benign tumors of the esophagus are rare - found in $0.005-7.9 \%$ of cases and leiomyomas account for 70 to $80 \%$ of these tumors $[2,3]$. Most cases are diagnosed in adults aged between 20 to 80 years, with the majority of the patients being between 40 and 50 years [4]. At least half of the patients are asymptomatic and are often diagnosed incidentally during esophagogastroduodenoscopy (EGDS). The most common symptoms are dysphagia, pain and weight loss [1, 5]. The diagnosis usually requires a combination of imaging and endoscopic methods such as EGDS, computer tomography (CT), magnetic resonance imaging (MRI), endoscopic ultrasound (EUS), contrast esophagogram. Because of their subepithelial localization in the esophageal wall, tissue biopsies during EGDS are often falsenegative (even if the bite on bite technique is utilized). Treatment of symptomatic cases and large tumors without definite histological characterization is surgical. The novel endoscopic or combined techniques may be applied in selected patients [610].

\section{Case report:}

40-years old male patient complained of mild episodic dysphagia, mainly for solid foods in the last six months. There was no significant weight loss during the period. The patient had L4-L5-S1 hernia. There were no significant laboratory changes. In the department of gastroenterology, the patient underwent EGDS. We observed a large area of compression of the esophagus with no visible changes of the overlying mucosa. In the stomach, mild erosive antral gastritis was seen. 
At CT we found a well defined, ovoid tumor located in the posterior mediastinum at the level of the bifurcation of the trachea (Figure 1 and 2). The lesion was $36 \times 55 \times 75 \mathrm{~mm}$ in size. It was causing compression of the esophagus with associated proximal dilatation of the lumen. The native density of the lesion was $35 \mathrm{HU}$ and demonstrated no significant enhancement after contrast agent application. No demarcated capsule or mural nodules were seen.

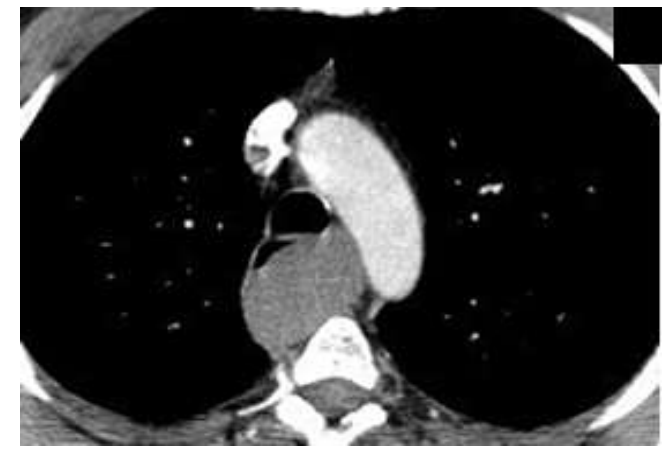

Figure 1. CT scan of esophageal leiomyoma at the level of the bifurcation of the trachea

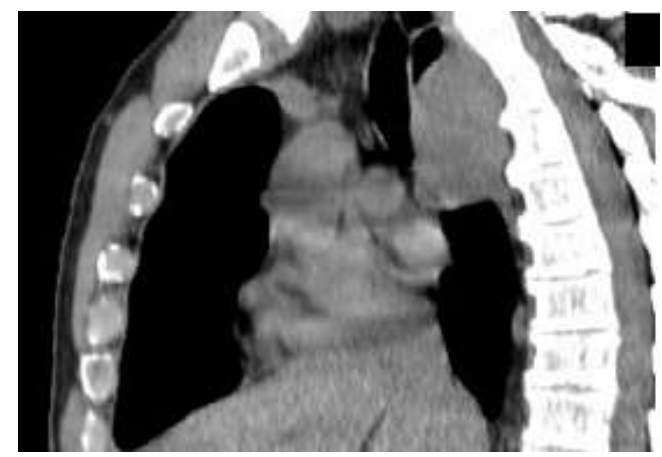

Figure 2. CT scan of esophageal leiomyoma at the level of the bifurcation of the trachea

EUS with linear echoendoscope was performed and demonstrated that the lesion was a subepithelial hypoechoic, heterogeneous solid tumor originating from the 4th layer of the wall - muscularis propria with an approximate size of 70x50 mm (Figure 3).

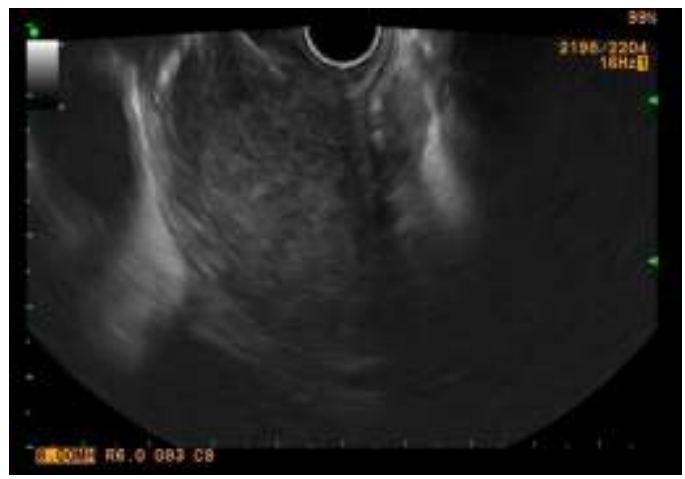

Figure 3. EUS image demonstrating a heterogenous hypoechoic subepithelial tumor arising from the 4th layer (muscularis propria) of the wall of the esophagus
On the basis of the imaging studies, a decision for surgical treatment was taken. A right-sided lateral minithoracothomy was performed. A solid tumor $75 \times 55 \mathrm{~mm}$ in size arising from the wall of the upper third of the esophagus was found. The right wall of the esophagus was dissected in its upper third

(Figure 4).

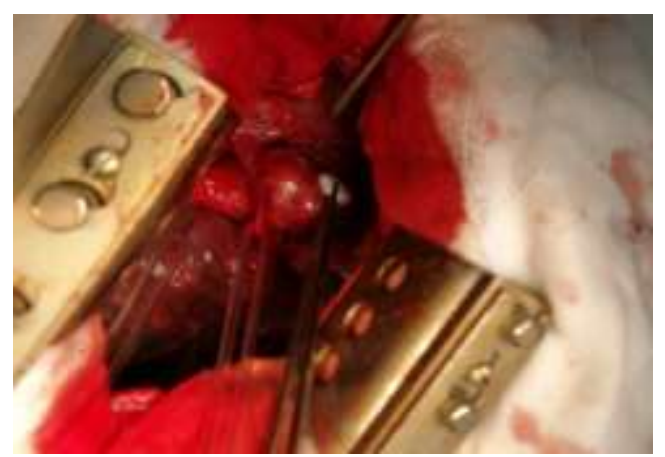

Figure 4. Dissection of the medial wall of the esophagus in the area of the tumor from the mediastinal pleura and azigos vein

Its longitudinal and circular layers were incised together with the overlaying adventitia, and the lesion was reached. The tumor was carefully dissected and enucleated from the thinned up mucosa (Figure 5 and 6). The muscle layer and adventitia were sawed up with interrupted 000 stitches.

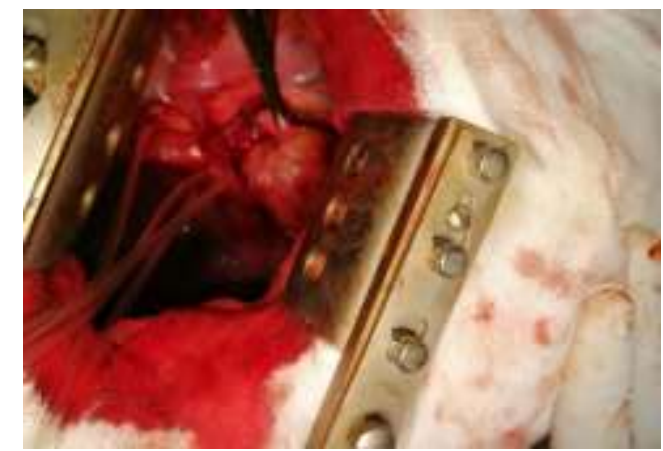

Figure 5. Enucleation of the tumor

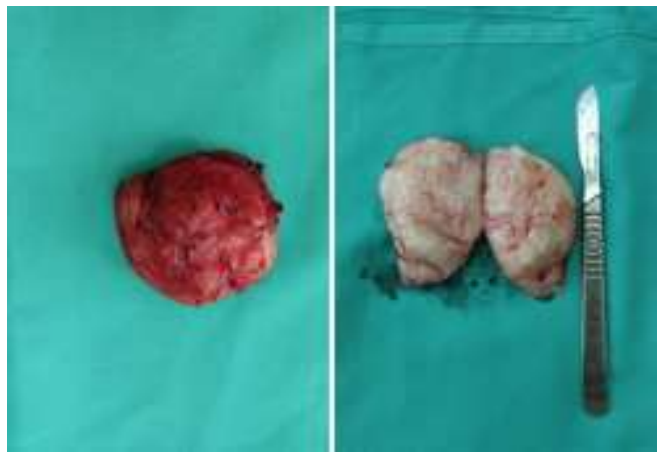

Figure 6. Surgically removed leiomyoma 


\section{Discussion:}

Leiomyomas are the most common benign tumors of the esophagus and can be found in any of its parts. About $60 \%$ are situated in the distal third of the esophagus, about $30 \%$ in the middle third and $10 \%$ in the proximal [7]. In some cases, the preoperative diagnosis might be uncertain as parts of the patients are misdiagnosed with a mediastinal tumor. The combination of diagnostic modalities such CT, contrast esophagography, EGDS and EUS can facilitate the correct diagnosis [8]. The differential diagnosis with GIST is of great importance as GISTs have malignant potential and may require a different therapeutic approach $[9,10]$. Under EUS control, morphological verification using either EUS fine-needle aspiration (EUS-FNA) or EUS fine needle biopsy (EUS-FNB) can be performed. Depending on the technique and used equipment, biopsies may not yield sufficient material for histologic diagnosis in 20 to $50 \%$ of the cases. Malignant transformation of leiomyomas in leiomyosarcomas is extremely rare and is reported in less than $1 \%$. Combined histologic variants of leiomyoma and esophageal adenocarcinoma are described $[4,11]$. The therapeutic approach depends on the size, location of the tumor as well as the presence of symptoms, the age of the patient or any comorbidity. Small asymptomatic, histologically confirmed tumors might be observed. Observation is also sometimes advised in tumors less than $2 \mathrm{~cm}$ in size without histological verification, as there is a low risk of malignancy and EUS-FNA and EUSFNB of such lesions is inconclusive in a large proportion of the cases. If the diagnosis is uncertain, especially in large tumors, operative treatment is advised due to the malignant potential of other subepithelial tumors of the esophagus (especially GISTs). Surgical removal of the tumor can be achieved trough thoracoscopic or open (thoracotomy) surgical approach and enucleation of the tumor in the majority of patients. Nowadays, thoracoscopic enucleation is the gold standard [3, 12]. Recently endoscopic techniques for the extirpation, especially in tumors less than $2 \mathrm{~cm}$ in size are being used more widely due to less complication, faster postoperative recovery and decreased hospital stay $[6,13]$. A combined endoscopic and thoracoscopic approach has been described, which might be applicable in complicated cases [2]. Studies-suggested indications for open surgery are failure of singlelung ventilation, two large leiomyomas or intraoperative diagnosis of malignancy [14].
Resection is recommended for lower-third esophageal leiomyomas [15].

In our case, because of the size of the tumor an open surgery with minithoracotomy and enucleation of the tumor was chosen. In cases of large tumors, the separation of the tumor from the thin layer of mucosa might be difficult, and any laceration during the dissection might cause severe complications in the postoperative period. The tumor was carefully removed with preservation of the integrity of the mucosa.

\section{Conclusion:}

Leiomyomas of the esophagus are benign tumors with very favorable prognosis. Large and symptomatic tumors, especially lacking histologic verification, are candidates for surgical treatment. The choice of best therapeutic approach depends on a multitude of factors and should be made on a case-by-case basis.

\section{References:}

[1.] Kohli DR, Faigel DO. Esophageal leiomyomas: Making mole hills out of mole hills? Gastrointest Endosc. 2018;87(2):378379. doi:10.1016/j.gie.2017.08.028

[2.] Oyama K, Ohuchida K, Shindo K, et al. Thoracoscopic surgery combined with endoscopic creation of a submucosal tunnel for a large complicated esophageal leiomyoma. Surg Case Rep. 2020;6(1):92. Published 2020 May 6. doi:10.1186/s40792020-00854-5

[3.] Xu H, Li Y, Wang F, Wang W, Zhang L. Video-Assisted Thoracoscopic Surgery for Esophageal Leiomyoma: A Ten-Year SingleInstitution Experience. J Laparoendosc Adv Surg Tech. 2018; 28(9):1105-1108. doi:10.1089/lap.2018.0412

[4.] Sun LJ, Chen X, Dai YN, et al. Endoscopic ultrasonography in the diagnosis and treatment strategy choice of esophageal leiomyoma. Clinics. 2017; 72(4):197-201. doi:10.6061/clinics/2017(04)01

[5.] Jiang W, Rice TW, Goldblum JR. Esophageal leiomyoma: Experience from a single institution. Dis Esophagus. 2013; 26(2):167174. doi:10.1111/j.1442-2050.2012.01345.x

[6.] Tang X, Tan Y, Lv L, Liu D. Submucosal tunneling endoscopic resection for seven esophageal leiomyomas. Endoscopy. 2018; 50(3):E59-E60. doi:10.1055/s-0043-123821 
[7.] Peters JH, De Meester TR. Esophagus and diaphragmatic hernia. In: Brunicardi FC, Andersen KD, Biliar RT, Dunn LD, Hunter GC, Pollock RE, eds. Schwartz's Principle of Surgery. 8th Ed. New York: McGraw-Hill, 2005; 906.

[8.] Luh SP, Hou SM, Fang CC, Chen CY. Videothoracoscopic enucleation of esophageal leiomyoma. World J Surg Oncol. 2012;10:52. Published 2012 Mar 16. doi:10.1186/14777819-10-52

[9.] Punpale A, Rangole A, Bhambhani N, et al. Leiomyoma of esophagus. Ann Thorac Cardiovasc Surg. 2007; 13:78-81.

[10.] Wang YX, Zhang J, Liu Y, Liu Y, Chu XY, Lu ZS, Wang ZB, Tong XY. Diagnosis and comprehensive treatment of esophageal leiomyoma: clinical analysis of 77 patients. Int J Clin Exp Med. 2015;8(10):17214-17220.

[11.] FU KI, Muto M, Meta K, et al. Carcinoma coexisting with esophageal leiomyoma. Gastrointest Endoscopy. 2002; 56:272-273.

[12.] Jiang G, Zhao H, Yang F, Li J, Li Y, Liu J, Wang J. Thoracoscopic enucleation of esophageal leiomyomas: a retrospective study on 40cases. Dis esophagus. 2009;22(3):279283

[13.] Kinney T, Waxman I. Treatment of benign esophageal tumors by endoscopic techniques. Semin Thorac Cardiovasc Surg. 2003; 15(1):27-34.

[14.] Shin S, Choi YS, Shim YM, Kim HK, Kim K, Kim J. Enucleation of esophageal submucosal tumors: A single institution's experience. Ann Thorac Surg. 2014; 97:454-459.

[15.] Asteriou C, Konstantinou D, Lalountas M, et al. Nine years experience in surgical approach of leiomyomatosis of esophagus. World $\mathrm{J}$ Surg Oncol. 2009; 7:102. Published 2009 Dec 23. doi:10.1186/1477-7819-7-102 